(C) 2006 IEEE. Personal use of this material is permitted. Permission from IEEE must be obtained for all other uses, in any current or future media, including reprinting/republishing this material for advertising or promotional purposes, creating new collective works, for resale or redistribution to servers or lists, or reuse of any copyrighted component of this work in other works. 


\title{
Calibration of Sensing Coils of a Three- dimensional Magnetic Property Tester
}

\author{
Youguang Guo, Jianguo Zhu, Zhiwei Lin, Jinjiang Zhong, Haiwei Lu, and Shuhong Wang
}

\begin{abstract}
For electrical machines with three-dimensional (3D) magnetic fluxes, the magnetic properties of magnetic materials under 3D excitations should be properly determined and applied in machine design and analysis. This paper presents the construction and calibration of the $H$ (field strength) and B (flux density) sensing coils of a 3D magnetic property testing system, and the correction of measurement error due to the misalignment of sensing coils with excitation fields. The 3D tester has been used to measure the magnetic properties of soft magnetic composite (SMC), a material specially developed for application of 3D flux electrical machines. Some $3 D$ results have been obtained on a cubic SMC sample. The measurement errors caused by the misalignment of sensing coils and excitation fields are corrected by a rotational transformation of coordinates.
\end{abstract}

Index Terms-3D magnetic property tester, sensing coils, calibration, misalignment, rotational transformation of coordinates.

\section{INTRODUCTION}

$\mathrm{I}$ $\mathrm{N}$ MANY situations, such as the end regions of rotating 1 machines, and some machines with special topologies, e.g. claw pole/transverse flux motors, the magnetic flux is not restricted within a two-dimensional (2D) plane, and hence the magnetic properties of materials under three-dimensional (3D) magnetic excitations should be determined and properly applied in the motor design and performance analysis [1, 2]. As a matter of fact, in a magnetic material even with an alternating (one dimensional or $1 \mathrm{D}$ ) or 2D rotating magnetic field, the magnetic flux is 3D due to the rotation of magnetic domains. To investigate the magnetic properties of magnetic materials under 3D flux, a 3D magnetic tester has been developed by the authors [3]. To achieve accurate measurements, proper calibration of the $\mathbf{H}$ (field strength) and B (flux density) sensing coils are crucial.

This paper reports our work on the construction, calibration and measurement error correction of the sensing coils of the 3D magnetic testing system. Some measurements have been conducted on a cubic sample of SOMALOY $500[4,5]$, a soft magnetic composite (SMC) material specially developed

This work was supported by an Australian Research Council Large Grant (A00104148).

Y.G. Guo, J.G. Zhu, Z.W. Lin, J.J. Zhong, and H.W. Lu are with the Faculty of Engineering, University of Technology, Sydney, PO Box 123, Broadway, NSW 2007, Australia (e-mail: youguang.guo-1@uts.edu.au).

S.H. Wang is with the Faculty of Electrical Engineering, Xi'an Jiao Tong University, Xi'an, 710049, China. for electromagnetic devices with complex structure and 3D fluxes, due to the material's unique property such as magnetic and thermal isotropy. The misalignment between the sensing coil and the excitation field, which may cause measurement error, is corrected by a rotational transformation of coordinates. Some 3D results are presented and discussed.

\section{3D Magnetic PRoperty Tester}

Fig. 1 illustrates the block diagram of the 3D magnetic property testing system, and Fig. 2 shows the tester magnetic circuit. The system consists of a 3D yoke to guide the magnetic fluxes along three orthogonal directions, a data acquisition system, three groups of excitation coils which are wound around the legs of the 3D yoke, and a feedback control system comprising a control unit and three high power amplifiers. A cubic sample of the tested magnetic material is placed at the center between the poles of the yoke. For the material with isotropic magnetic properties, e.g. SMC, the testing sample can be made of a solid cube.

By controlling the magnetic excitations in three axes, the tester is able to generate different $\mathbf{B}$ patterns, such as alternating in any given orientation, rotating in a plane tilted for a given angle from any axis, and rotating in a 3D pattern with the loci of the $\mathbf{B}$ vector tip forming an ellipsoidal or spherical surface, according to the requirement of the test.

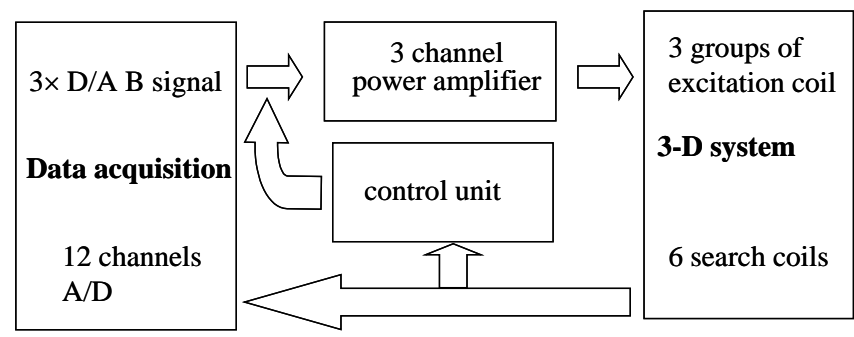

Fig. 1. 3D magnetic property testing system

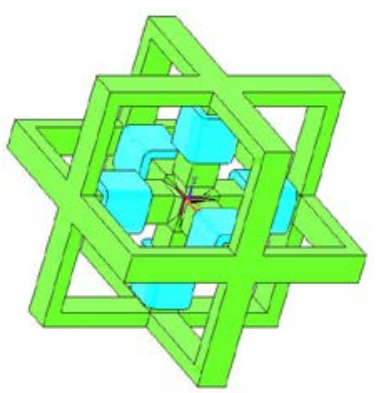

(a)

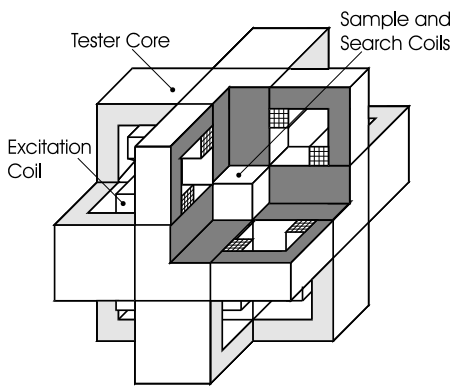

(b)
Fig. 2. Structure of 3D magnetic tester, (a) framework, and (b) cut away view 


\section{CONSTRUCTION AND CALIBRATION OF SENSING COILS}

Fig. 3 shows a sample with three $\mathbf{B}$ coils and a 3D $\mathbf{H}$ sensor unit. The $\mathbf{B}$ coils are wound directly around the sample. The 3D $\mathbf{H}$ sensor consists of six preformed 2D $\mathbf{H}$ sensing coils, which are placed inside a $36 \times 36 \times 36 \mathrm{~mm}$ cubic box made of 1 $\mathrm{mm}$ thick printed circuit boards. A $2 \mathrm{D} \mathbf{H}$ sensing coil is a group of two coils wound together on a $25 \times 25 \times 0.7 \mathrm{~mm}$ plastic former with their sensing axes perpendicular to each other. For each axis, there are four $\mathbf{H}$ coils, and these surface $\mathbf{H}$ coils are connected in series to give an average value of $\mathbf{H}$ components.

The measured $\mathrm{B}$ is the average value over the cross section of the sample. When there is no surface current, the tangential $\mathbf{H}$ components just outside and inside the surface of the sample have the same magnitude. The $\mathbf{B}$ and $\mathbf{H}$ components can be obtained by

$$
\begin{aligned}
& \mathrm{B}_{\mathrm{i}}=\frac{1}{\mathrm{~K}_{\mathrm{Bi}}} \int \mathrm{V}_{\mathrm{Bi}} \mathrm{dt} \\
& \mathrm{H}_{\mathrm{i}}=\frac{1}{\mu_{0} \mathrm{~K}_{\mathrm{Hi}}} \int \mathrm{V}_{\mathrm{Hi}} \mathrm{dt}
\end{aligned}
$$

where $i=\mathrm{X}, \mathrm{Y}, \mathrm{Z}, \mathrm{K}_{\mathrm{Bi}}=\mathrm{N}_{\mathrm{Bi}} \mathrm{h}_{\mathrm{sp}} \mathrm{w}_{\mathrm{i}}$ is the coefficient of the $\mathbf{B}$ sensing coil, $\mathrm{N}_{\mathrm{Bi}}$ the number of turns of the $\mathbf{B}$ sensing coil, $\mathrm{h}_{\mathrm{sp}}$ the height of the sample, $w_{\mathrm{i}}$ the width of sample, $\mathrm{K}_{\mathrm{Hi}}$ the $\mathbf{H}$ sensing coil coefficient obtained by calibration, and $\mathrm{V}_{\mathrm{Bi}}$ and $\mathrm{V}_{\mathrm{Hi}}$ are terminal voltages of the $\mathbf{B}$ and $\mathbf{H}$ sensing coils.

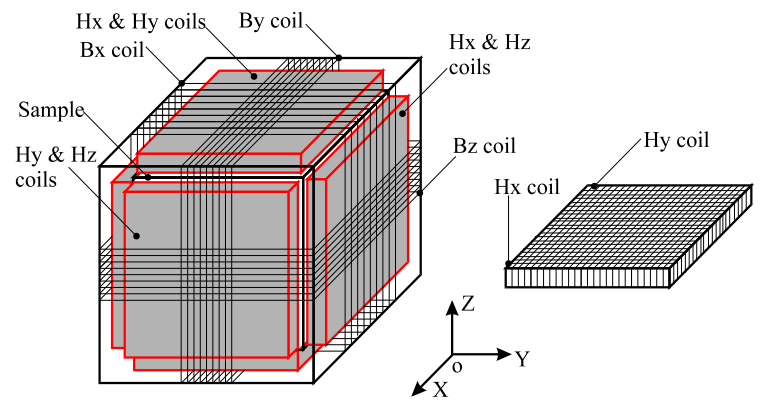

Fig. 3. $\mathbf{B}$ and $\mathbf{H}$ sensing coils on a cubic sample

The 3D $\mathbf{H}$ sensor is calibrated in a solenoid as shown in Fig. 4, where $\mathrm{A}$ is the solenoid frame, B the $\mathbf{H}$ coils to be calibrated, C the Gauss meter probe, D the connector for the output terminals of sensing coil, E the turntable for mounting the coils, and $\mathrm{F}$ and $\mathrm{G}$ are the wheel and the rubber band for adjusting the coil orientation. The solenoid is excited with a sinusoidal current. The flux density in the center of the solenoid is uniform and proportional to the excitation current. It is calculated from the magnitude of the excitation current and double checked by a high precision digital Gauss meter. The sensing coils to be calibrated are mounted on a small turntable. When the output voltage signal of the coils on one axis, which is proportional to the excitation frequency and the flux density in the center of the solenoid, reaches the maximum, the axis of these coils is aligned with the solenoid axis. The coil coefficient can be determined by

$$
\mathrm{K}_{\mathrm{H}}=\frac{\mathrm{V}_{\mathrm{H}}}{\sqrt{2} \pi \mathrm{f} \mu_{0} \mathrm{H}_{\mathrm{m}}}
$$

where $V_{H}$ is the rms value of the sensing coil terminal voltage when the coil axis is aligned with the field, $f$ the frequency of the excitation current, and $\mathrm{H}_{\mathrm{m}}$ the magnitude of the magnetic field strength in the center of the solenoid. The results are averaged over a number of measurements as $\mathrm{K}_{\mathrm{Hx}}=0.044960$, $\mathrm{K}_{\mathrm{Hy}}=0.039603$, and $\mathrm{K}_{\mathrm{Hz}}=0.040557$. The calibration of $\mathbf{B}$ coils is done in the same way.

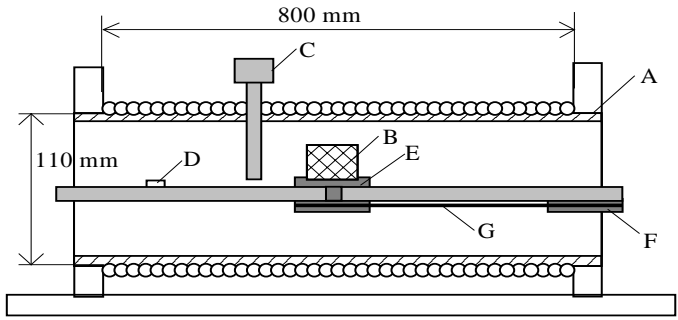

Solenoid: $\mathrm{N}=939$ (turns), and H/I=1162.8 (1/m)

Fig. 4. Calibration of $\mathbf{H}$ sensing coils in a solenoid

\section{CORRECTION OF ERROR DUE TO MisALignMENT OF SENSING COILS BY ROTATION OF COORDINATES}

As in 2D measurements, when the axes of the sensing coils are misaligned with the axes of the excitation coils, the measured values are not the true components on their axes of the magnetic field strength or flux density [6]. Theoretically, this error can be corrected by using the rotational transformation of coordinates. In the 3D magnetic tester, the error caused by the sensing coil misalignment can also be eliminated by a rotation of the coordinate axes. However, the misalign angles cannot be readily obtained from the measured field components.

Fig. 5 illustrates the rotation of coordinates in three dimensions where $\mathrm{X}, \mathrm{Y}$, and $\mathrm{Z}$ are orthogonal coordinate axes of the measurement system. $\mathrm{X}^{\prime}, \mathrm{Y}^{\prime}$, and $\mathrm{Z}^{\prime}$ are misaligned axes of the 3D sensor. The angles between $\mathrm{X}^{\prime}$ axis and $\mathrm{X}, \mathrm{Y}$ and $\mathrm{Z}$ axes are denoted as $\theta_{1}, \phi_{1}$ and $\psi_{1}$, respectively. Similarly, the angles between $\mathrm{Y}^{\prime}$ and $\mathrm{X}, \mathrm{Y}, \mathrm{Z}$ and $\mathrm{Z}^{\prime}$ and $\mathrm{X}, \mathrm{Y}, \mathrm{Z}$ are $\theta_{2}, \phi_{2}$, $\psi_{2}$, and $\theta_{3}, \phi_{3}, \psi_{3}$, respectively.

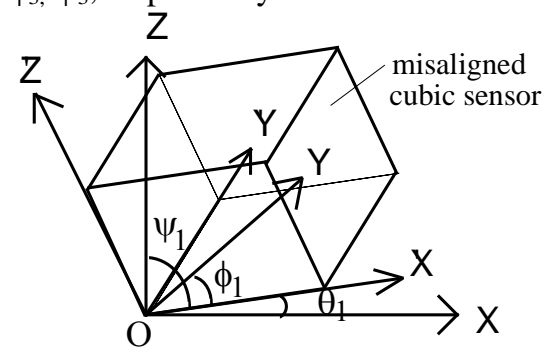

Fig. 5. Misalignment of sensor and rotation of coordinates

According to the rotational transformation, we have

$$
\left[\begin{array}{l}
H_{x}^{\prime} \\
H_{y}^{\prime} \\
H_{z}^{\prime}
\end{array}\right]=\left[\begin{array}{ccc}
\cos \theta_{1} & \cos \phi_{1} & \cos \psi_{1} \\
\cos \theta_{2} & \cos \phi_{2} & \cos \psi_{2} \\
\cos \theta_{3} & \cos \phi_{3} & \cos \psi_{3}
\end{array}\right]\left[\begin{array}{l}
H_{x} \\
H_{y} \\
H_{z}
\end{array}\right]
$$

where $\mathrm{H}_{\mathrm{x}}{ }^{\prime}, \mathrm{H}_{\mathrm{y}}{ }^{\prime}$, and $\mathrm{H}_{\mathrm{z}}{ }^{\prime}$ are the measured component values of the magnetic field strength, and $\mathrm{H}_{\mathrm{x}}, \mathrm{H}_{\mathrm{y}}$, and $\mathrm{H}_{\mathrm{z}}$ the true values of magnetic field strength.

In order to evaluate the angles $\theta_{\mathrm{i}}, \phi_{\mathrm{i}}, \psi_{\mathrm{i}}(\mathrm{i}=1,2,3)$, excitation fields in $\mathrm{X}, \mathrm{Y}$, and $\mathrm{Z}$ axes are applied respectively. 
When an excitation field is applied only in $\mathrm{X}$ or $\mathrm{Y}$ or $\mathrm{Z}$ axis, the field components $\mathrm{H}_{\mathrm{x}}{ }^{\prime}, \mathrm{H}_{\mathrm{y}}{ }^{\prime}$, and $\mathrm{H}_{\mathrm{z}}{ }^{\prime}$ can be measured by the $\mathbf{H}$ sensing coils. We denote the projection angle of the misaligned axis $\mathrm{X}^{\prime}$ in XOY as $\theta_{\mathrm{x} 1}$, and $\theta_{\mathrm{x} 2}$ in XOZ. By using the method for 2D measurement, the values of the projection angles $\theta_{\mathrm{x} 1}$ and $\theta_{\mathrm{x} 2}$ can be calculated. Thus, the angle $\theta_{1}, \phi_{1}$ and $\psi_{1}$ can be calculated by

$$
\begin{aligned}
& \theta_{1}=\tan ^{-1}\left(\sqrt{\tan ^{2} \theta_{x 1}+\tan ^{2} \theta_{x 2}}\right) \\
& \phi_{1}=\tan ^{-1}\left(\frac{1}{\cos \theta_{x 2} \tan \theta_{x 1}}\right) \\
& \psi_{1}=\tan ^{-1}\left(\frac{1}{\cos \theta_{x 1} \tan \theta_{x 2}}\right)
\end{aligned}
$$

Similarly, we denote the projection angle of the misaligned axis $\mathrm{Y}^{\prime}$ as $\phi_{\mathrm{y} 1}$ in $\mathrm{XOY}$, and $\phi_{\mathrm{y} 2}$ in YOZ. The misalignment angles $\theta_{2}, \phi_{2}$ and $\psi_{2}$ can be expressed by

$$
\begin{aligned}
& \theta_{2}=\tan ^{-1}\left(\frac{1}{\cos \phi_{y 2} \tan \phi_{y 1}}\right) \\
& \phi_{2}=\tan ^{-1}\left(\sqrt{\tan ^{2} \phi_{y 1}+\tan ^{2} \phi_{y 2}}\right) \\
& \psi_{2}=\tan ^{-1}\left(\frac{1}{\cos \phi_{y 1} \tan \phi_{y 2}}\right)
\end{aligned}
$$

If the projection angles of the misaligned axis $\mathrm{Z}^{\prime}$ are denoted as $\psi_{\mathrm{z} 1}$ in $\mathrm{XOZ}$ and $\psi_{\mathrm{z} 2}$ in $\mathrm{YOZ}$, the misalignment angles $\theta_{3}, \phi_{3}$ and $\psi_{3}$, for $Z^{\prime}$ can be determined by

$$
\begin{aligned}
& \theta_{3}=\tan ^{-1}\left(\frac{1}{\cos \psi_{z 2} \tan \psi_{z 1}}\right) \\
& \phi_{3}=\tan ^{-1}\left(\frac{1}{\cos \psi_{z 1} \tan \psi_{z 2}}\right) \\
& \psi_{3}=\tan ^{-1} \sqrt{\tan ^{2} \psi_{z 1}+\tan ^{2} \psi_{z 2}}
\end{aligned}
$$

Then, the true values of the surface magnetic field strength $\mathbf{H}$ can be evaluated from the measured values by (4) - (7).

\section{3D Magnetic MeAsurement}

The 3D magnetic tester has been used to measure the magnetic property of SOMALOY ${ }^{\mathrm{TM}} 500$. Three B search coils wounded around the sample provide signals of three direction components of the $\mathbf{B}$ vector, while the $\mathbf{H}$ is measured with the calibrated surface $\mathbf{H}$ search coils placed on the six surfaces of the cubic specimen. In this way, the B-H loops are obtained. The corresponding core losses can be worked out according to the Poynting's theorem by

$$
P_{t}=\frac{1}{T \rho_{m}} \int_{0}^{T}\left(H_{x} \frac{d B_{x}}{d t}+H_{y} \frac{d B_{y}}{d t}+H_{z} \frac{d B_{z}}{d t}\right) d t
$$

where $P_{t}$ is the specific core loss (in $\mathrm{W} / \mathrm{kg}$ ) in the sample due to a time-varying $3 \mathrm{D} \mathbf{B}$ vector, $T=1 / f$ is the time period, $f$ the frequency of magnetization, and $\rho_{m}$ the sample mass density.

Three groups of computer controlled excitation coils are employed to generate various $\boldsymbol{B}$ patterns including the ones in real 3D space, such as the patterns with the locus of the $\boldsymbol{B}$ vector tip forming an ellipsoidal or spherical surface, as illustrated in Fig. 6. The measurement errors due to the misalignment of sensing coils are corrected by the rotational transformation of coordinates.

Other measured results include the B-H and core loss curves when the $\mathbf{B}$ vector is controlled as $1 \mathrm{D}$ alternating along the $\mathrm{X}$, $\mathrm{Y}$, or $\mathrm{Z}$ axis, or $2 \mathrm{D}$ circularly or elliptically rotating in the XOY, YOZ, or ZOX planes, respectively, etc. It is noted that these measurements are different from those obtained by conventional $1 \mathrm{D}$ and $2 \mathrm{D}$ testers as the latter cannot observe the effect of the third direction. The measured results have been applied in the design and analysis of a claw pole/transverse flux motor with SMC core [2].

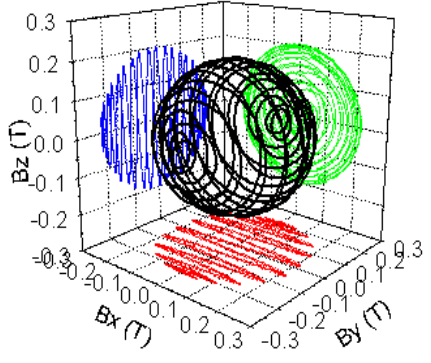

(a)

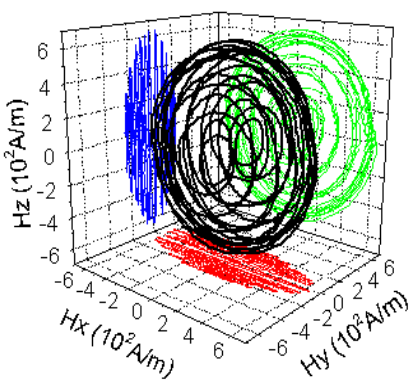

(b)
Fig. 6. (a) $\mathbf{B}$ locus; and (b) corresponding $\mathbf{H}$ locus when the $\mathbf{B}$ locus is controlled to form a spherical surface

\section{CONCLUSION}

To investigate the magnetic properties of materials under 3D flux, a 3D magnetic tester has been developed. This paper presents the construction, calibration and measurement error correction of sensing coils of the magnetic tester. The calibrated magnetic tester has been used to measure the 3D magnetic properties of a cubic SMC sample.

\section{REFERENCES}

[1] J.G. Zhu, J.J. Zhong, Z.W. Lin, and J.D. Sievert, "Measurement of magnetic properties under 3-D magnetic excitations," IEEE Trans. Magn., Vol.39, No.5, Sept. 2003, pp. 3429-3431.

[2] Y.G. Guo, J.G. Zhu, Z.W. Lin, and J.J. Zhong, "Measurement and modeling of core losses of soft magnetic composites under 3D magnetic excitations in rotating motors," IEEE Trans. Magn., Vol.41, No.10, Oct. 2005, pp. 3925-3927.

[3] J.J. Zhong, "Measurement and modeling of magnetic properties of materials with rotating fluxes," Ph.D. Thesis, Univ. Technology, Sydney, Dec. 2002.

[4] "Soft magnetic composites from Höganäs Metal Powders SOMALOY ${ }^{\mathrm{TM}}$ 500,” Höganäs Product Manual, 1997.

[5] "The latest development in soft magnetic composite technology," SMC Update, Reports of Höganäs AB, Sweden, 1997-2005. Available at http://www.hoganas.com/, see News then SMC Update.

[6] J.G. Zhu, "Numerical modeling of magnetic materials for computer aided design of electromagnetic devices," Ph.D. Thesis, Univ. Technology, Sydney, July 1994. 